\title{
A Novel Surface Completion Method
}

\author{
Zhen $\mathrm{Liu}^{1, \mathrm{a}}{ }^{*}$, Li Li Bai, ${ }^{2, \mathrm{~b}}$ and Yong Wei Miao, \\ ${ }^{1,2}$ College of Science, Zhejiang University of Technology, China \\ ${ }^{3}$ College of Computer Science and Technology, Zhejiang University of Technology, China

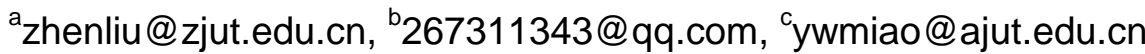

Keywords: Surface completion; Fitted quadric surface; Surface details; Base surface; MAFM.

\begin{abstract}
Surface completion is a classical and significant research topic in the area of digital geometry processing. Due to the expensive time consumption and the unreliability of the existing popular algorithm, a surface completion method is proposed in this paper that can recover the significant geometric information of the underlying model. Firstly, the hole is filled by a smooth patch by modified advancing front mesh technique. Secondly, the fine-scaled details are added by fitting quadric surface from the boundary of the hole to its center. The experimental results demonstrate that our surface completion scheme can effectively fill the holes and recover the fine-scale details of the hole region.
\end{abstract}

\section{Introduction}

Digital 3D models are the one of the most important part in numerous industrial and scientific sectors. However, due to limitations of the acquiring model technologies or incompletion of the source objects, the obtained models often contain holes of missing geometry and cannot be directly utilized in the subsequent geometry processing. Thus, it is desirable to deal with the errors on a polygonal model, such as gaps, holes, non-manifold elements or self-intersections for the applications that require certain quality model as input. In this paper, we consider the problem of holes in the input model.

There are many hole filling approaches that have been presented in the literature of digital geometry processing. Each type method has its limits, and even a good completion on the setting could perform undesired result on the other settings. Early hole-complete algorithms consider about how to triangulate the hole region, such as guided by minimal areas [1], minimal distances [2] or angle measures [3]. However, it's hard to get an ideal effect for the holes covering prominent geometric features. More recent hole-filling approaches focus on recovering the detail on the surface and make it as close as possible to the original shape of 3D model. These methods will recover the missing geometric features rely on the regions which makes it has similar geometry on the input model or some other existing models. This kind of methods can produce a compelling rendition of the complete geometry if the similar geometry can be found. However, the time complexity of example-based methods is usually very high. We are aim to propose a method that can recover prominent geometric features and irrelevant with whether there is similar geometry on input mesh or not. Here, we employ the least squares method to fitting surfaces so as to add fine-scaled details to the hole region. Our main contributions of this paper are as follows.

The holes in the model are filled by some smooth patches with our pre-process based on the modified advancing front mesh (MAFM) technique.

The fine-scaled details are added in the order which is from the boundary of the hole to its center. And the geometric details are recovered by projecting the vertices in the hole region to their corresponding fitted quadric surface. 


\section{Related Work}

Filling hole is a long history question, and many methods had been proposed. Ju [4] did a comprehensive survey about surface completion and classified the current hole filling techniques into two main directions: based on volume or based on surface.

Volume-based methods convert the input mesh into volumetric grid, after repairing then using different isosurface extraction method to recover mesh model. Davis et al.[5] design a signed distance function over volumetric grid and then use diffusion to propagated to the whole volumetric grid field. Nooruddin and Turk [6] proposed a volume-based technical to transform the input model into a uniform adaptive sampling distance field, and then polygonize into a simplified version of the input model which can repair arbitrary mesh, but it could not deal with the sharp features. Ju [7] put forward a method to repair on dual mesh, which is fit for arbitrary large mesh. Bischoff and Kobbelt [8] built an adaptive octree based on input mesh.

Surface-based technology directly detection the holes and the surface geometry and focus on the hole area, keeping the rest part consist to the original model. Classical methods can be classified into two types. First type methods focus on how to repair the hole efficiently, such as triangulation and scattered data fitting techniques. Barequet and Kumar [9] stitch the corresponding edges to repair the cracks of the model. Liepa [10] triangulates the polygon after delimiting the hole, and then remesh to improve the quality. Verdera et al. [11] use implicit surface to define the repair area, and defines a normal vector field based partial differential equation to interpolation holes' boundary smoothly. Zhao et al. [12] precede a rough completion by wavefront propulsion method and then optimization by adjusting the position of vertexes through poisson equations. Pernot et al. [13] inserting faces which keeping minimum curvature around the hole to fill the holes. Therefore, the second type of model repair methods considers more about geometrical details. Nguyen et al. [14] propose a method to synthesis details after smoothly repairing the holes. They transform three-dimensional geometric detail synthesis problem into a two-dimensional problem through parameterized. Sharf et al. [16] extend the example-based image inpainting algorithms to the point-to-point model. Example-based method could divide the repair work into two stage process [17], the underlying surface completion and the detail completion. Acqua and Fisher [18] provide some basic geometry to match the hole and the detail completion process is to propagation the localized surface relief, for uncomplete model. Use the input model as sample, and regard the hole as the target, searching the sample to fine the similar geometry to the target, and copy the local detail to the target [17]. Breckon and Fisher [19] also propose a hierarchical extension to the earlier approach to solve the presence of global discontinuities. Based the example-based idea, Harary et al. [20] introduce the coherent as object to make the result more compelling. These methods could acquire an ideal result if there have similar parts to the missing parts on the input mesh. However, they will use more time as cost.

\section{Base Surface Construction by MAFM}

We construct the base surface by the modified advancing front mesh (MAFM) technique [15] to generate an initial patch mesh in the hole region. Then the new added vertices in the base surface may be scraggly and sparse. Hence the patch mesh in the hole region cannot blend to the original surface smoothly. Therefore the mesh in the hole patch need to be refined and the vertices in the hole region should be repositioned so as to blend with the geometry of the surrounding mesh.

\section{Surface Completion}

In order to utilize the information of the surrounding mesh to recover the geometric details as much as possible, two steps are presented in this section. Firstly, a method of refinement is employed to make sure the vertices' density in the hole patch is the same as to the density of the surrounding mesh. Secondly, we present the least square fitted surface to recover the fine-scaled details in the hole patch. 


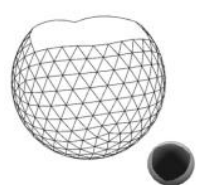

(a)

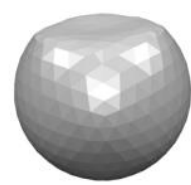

(b)

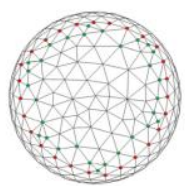

(c)

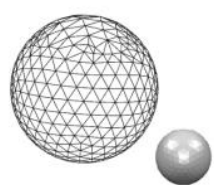

(d)

Figure. 2 Surface completion using our surface completion method.

(a): Original sphere model with hole; (b): The hole is filled by smooth patch which is constructed by the method of modified advancing front mesh; (c): The enlarged drawing of (b). The vertices with red color is the boundary of the original sphere model, and the vertices with green color is the one-ring vertices of the boundary in the hole patch; (d): The final completion result.

Pre-process of the Patch in the Hole Region. Because the new vertices on the initial patch mesh are ragged and scraggly, we use uniform discretization of the Laplace-Beltrami operator to smooth the hole patch [21]. The uniform Laplace-Beltrami operator is

$$
\Delta f\left(\mathbf{v}_{i}\right)=\frac{1}{\left|N_{1}\left(\mathbf{v}_{i}\right)\right|} \sum_{\mathbf{v}_{i} \in N_{1}\left(\mathbf{v}_{i}\right)}\left(f_{i}-f_{j}\right)
$$

where the sum is taken over all one-ring neighbors $\mathbf{v}_{i} \in N_{1}\left(\mathbf{v}_{i}\right)$. Applied to the coordinate function $x$, the uniform graph Laplacian $\Delta x_{i}$ evaluates to the vector pointing from the center vertex $x_{i}$ to the average of the one-ring vertices $x_{j}$. The uniform Laplace-Beltrami operator is efficient and easy to compute.

Then a method of refinement is used to refine the triangles in the hole patch. The method of refinement for a triangle $\Delta \mathbf{v}_{i} \mathbf{v}_{j} \mathbf{v}_{k}$ in the hole patch is that the triangle $\Delta \mathbf{v}_{i} \mathbf{v}_{j} \mathbf{v}_{k}$ will be split into three triangles $\Delta \mathbf{v}_{i} \mathbf{v}_{j} \mathbf{G}, \Delta \mathbf{v}_{i} \mathbf{v}_{k} \mathbf{G}$ and $\Delta \mathbf{v}_{j} \mathbf{v}_{k} \mathbf{G}$ if the average length of the triangle $\Delta \mathbf{v}_{i} \mathbf{v}_{j} \mathbf{v}_{k}$ is larger than the average length of the hole's boundary. Where $\mathbf{G}$ is the centroid of the triangle $\Delta \mathbf{v}_{i} \mathbf{v}_{j} \mathbf{v}_{k}$.

Details Added by Least Square Fitted Surface. The missing details in the hole can be considered as the extension of the surface around the holes' boundary. Hence we locate the vertices in the hole patch to the corresponding fitted quadric surface. The process is as follows.

Let $M_{\text {original }}$ denote the original model with holes, and $M_{\text {completion }}$ denote the patch in the hole region.

Firstly, the one ring of the surface boundary is identified as the current target vertices $L$ in the hole patch. In Fig. 2 (c), the current target set of vertices $L$ is the vertices with green color. For each vertex $\mathbf{v}_{i} \in L$, we projected it to $\mathbf{v}_{i}^{\prime}$ which is in the corresponding quadric surface, i.e.,

$$
\mathbf{v}_{i}^{\prime}=v_{i}+\alpha \cdot \mathbf{n}
$$

where $\mathbf{n}$ is the average normal of the triangles with vertex $\mathbf{v}_{i}$. The quadric surface is fitted by following method. Firstly, we present the regulation of outside (or inside) of a closed boundary loop firstly. If we collect the boundary vertices $P=\left\{\mathbf{v}_{1}, \mathbf{v}_{2}, \ldots, \mathbf{v}_{n}\right\}$ by anticlockwise, then the outside

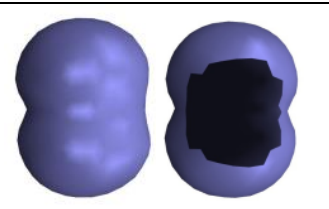

(a) (b)

Figure. 3 (a): Original hole; (b): Our result. (or inside) of the closed boundary loop $P$ is the vertices in the right (left) hand side. Secondly, the neighbor vertices of $\mathbf{v}_{i} \in L$ in the outside of the closed boundary loop are selected to fit the corresponding quadric surface.

Once all the vertices in $L$ are projected to the quadric surface, all the vertices in $L$ are belongs to $M_{\text {original }}\left(M_{\text {completion }}\right.$ becomes $\left.M_{\text {completion }} / L\right)$ and $L$ is the new boundary of the hole. Then we collect the one-ring of the vertices of the new boundary and project them to the fitted quadric surface until the set $M_{\text {completion }}$ becomes empty. Fig. 2 shows the pipeline of our surface completion method. And Fig. 3 shows an example of our surface completion. 


\section{Experimental Results and Discussion}

The tests are performed on a $2.3 \mathrm{GHz}$ Intel i3-processor with 4GB of memory. The running time, which depends on the size of the hole, is dozens of seconds. In our algorithm, we construct a smooth patch by modified advancing front mesh and fit quadric surface to add details. The holes are completed efficiently and whole time consumption is not too much. For instance, Fig. 4 show the model bunny with 33530 vertices, and the two holes are filled with 1250 vertices, the running time is no more than 40 seconds.

To illustrate the behavior of our algorithm, we show several examples where appealing completions of large holes are produced by our surface completion method. Then we compare our surface completion results to the existing hole filling approaches (see Fig. 3-6). In Fig. 4-6, (a) is original model, (b) is completed by Davis [5] , (c) is completed by Ju [7] and (d) is repaired by our method.

The advantage of our method is in its ability to propagate the fine-scale details in the hole's surrounding to the hole's inner efficiently. Fig. 3 is the completion of a ellipsoid with a little sunken in its middle part. Due to our vertices' reposition process is from the boundary of the hole to its center, the sunken part is propagated to the hole region naturally.

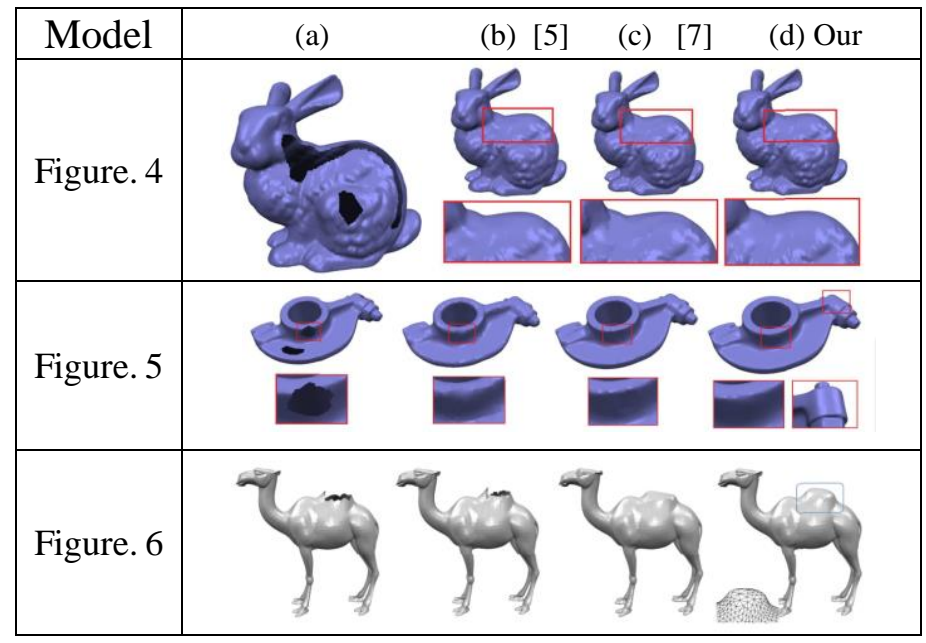

Fig. 4 shows t he completion of the bunny. Fig. 4(b) is the result of Davis method [5] and Fig. 4(c) is the result of $\mathrm{Ju}$ [7]. Both completions look pretty natural. Our method can reconstruct the fine-scale characteristics of 3D surfaces, such as the feature of the rabbit's neck. The back of the bunny is gentler, so we select their 4-rings to fit quadric surface. In our test, we select the 2-rings of the candidate points to fit least squares quadric surface if the corresponding curvature are lager than a threshold (In our experiments, it is 0.02.) and select their 4-rings if the corresponding curvature are smaller than the threshold on the contrary.

Our method can repair the holes of the model with arbitrary shape and avoid to break the original mesh, see Fig. 5. In Fig. 5, the broken rocker-arm has different size of holes. In our completion, the ridge is preserved and the original mesh is not changed. The completion result of Davis et al. approach [5] and Ju approach [7] show that the shape features in original model is destroyed. Fig. 6 shows another completion of the model camel. Compared with the existing hole filling approaches, our completion scheme can capture the detail information of the hump region and get better result.

\section{Conclusion}

In this paper, a surface completion method based on fitted quadric surface is presented to complete the holes of 3D surfaces. Our surface completion scheme can capture the fine-scale details in the hole patch efficiently. Experimental results show that our proposed approach can effectively recovery the surface details during hole filling. 
For some holes occur in practice, the missing of the object may not have similar patches or the finding process of the similar patches is time consuming. Compared with the other methods, our algorithm can improve the completion results of the models with holes which is hard to find similar patches. In the future, we would like to find the similar patches based on our method. Thus we will find the similar patch in a faster way for surface completion.

\section{References}

[1] G. Barequet and M. Sharir. Filling gaps in the boundary of a polyhedron. Computer-Aided Geometric Design, 1995, 12(2): 207-229.

[2] I. M" akel" a and A. Dolenc. Some efficient procedures for correcting triangulated models. In: Proceedings of Solid Free Form Fabrication Symposium, pages. 126-134, Austin, Texas, 1993.

[3] M.Varnuska, J.ParusandI.Kolingerova. Simple holes triangulation in surface reconstruction. In: Proceedings of ALGORITMY, pages 280-289, Vysoke Tatry, Podbanske, 2005.

[4] T. Ju. Fixing geometric errors on polygonal models: a survey. Journal of Computer Science and Technology, 2009, 24(1): 19-29.

[5] J. Davis, S.R. Marschner, M. Garr and M. Levoy. Filling holes incomplex surfaces using volumetric diffusion. In: Proceedings of Symposium on 3D Data Processing Visualization and Transmission (3DPVT), pages 428-438, Padova, Italy, 2002.

[6] F. S. Nooruddin and G. Turk. Simplification and repair of polygonal models using volumetric techniques. IEEE Transactions on Visualization and Computer Graphics, 2003, 9(2): 191-205.

[7] T. Ju. Robust repair of polygonal models. ACM Transactions on Graphics, 2004, 23(3): 888-895.

[8] S. Bischoff and L. Kobbelt. Structure preserving CAD model repair. Computer Graph Forum, 2005, 24(3): 527-536.

[9] G. Barequet and S. Kumar. Repairing CAD models. In: Proceedings of IEEE Visualization, pages 363-370, Phoenix, Arizona, 1997.

[10] P. Liepa. Filling holes in meshes. In: Porceedings of Eurographics/ACM SIGGRAPH Symposium on Geometry Processing, pages 200-205, Aachen, Germany, 2003.

[11] J. Verdera, V. Caselles, M. Bertalmio, and G. Sapiro. Inpainting surface holes. In: Proceddings of International Conference on Image Processing, pages 903-906, Barcelona, Spain, 2003.

[12] W. Zhao, S. Gao and H. Li. A robust hole-filling algorithm for triangular mesh. The Visual Computer, 2007, 23(12): 987-997.

[13] J. P. Pernot, G. Moraru and P. Veron. Filling holes in meshes using a mechanical model to simulate the curvature variation minimization. Computer and Graphics, 2006, 30(6): 892-902.

[14] M. Nguyen, X. Yuan and B. Chen. Geometry completion and detail generation by texture synthesis. The Visual Computer, 2005, 21(8): 669-678.

[15] W. Xiaochao, C. Junjie, L. Xiuping, L. Baojun. Advancing Front Method in Triangular Meshes Hole-Filling Application. Journal of Computer-Aided Design(Computer Graphics), 2011, 23(6): 1048 -

1054.

[16] A. Sharf, M. Alexa and D. Cohen-or. Context-based surface completion. ACM Transactions on Graphics, 2004, 23(3): 878-887.

[17] T. P. Breckon and R. B. Fisher. Three-dimensional surface relief completion via nonparametric techniques. IEEE Transactions on Pattern Analysis and Machine Intelligence, 2008, 30(12): 2249-2255.

[18] F. Dell' Acqua and R. Fisher. Reconstruction of planar surfaces behind occlusions in range images. IEEE Transactions on Pattern Analysis and Machine Intelligence, 2002, 24(4): 569-575.

[19] T. P. Breckon and R. B. Fisher. A hierarchical extension to 3D nonparametric surface relief completion. Pattern Recognition, 2012, 45(1): 172-185.

[20] G. Harary, A. Tal and E. Grinspun. Context-based coherent surface completion. ACM Transactions on Graphics, 2013, 32(3). 
[21] G. Taubin. Estimating the tensor of curvature of a surface from a polyhedral approximation. In: Proceedings of International Conference on Computer Vision, pages 902-907, Boston, Massachusetts, 1995. 